\title{
TRATAMENTO DA GIARDIASE EM CRIANÇAS PELA NITRIMIDAZINA
}

\author{
Donald Huggins *: e Marluce F. Maciel ***
}

\begin{abstract}
Os AA. trataram 30 crianças portadoras de giardiase com o derivado $\mathrm{Ni}$ trimidazinico - NAXOGINR, utilizado em suspensão contendo $200 \mathrm{mg}$ da substancia ativa por cada $5 \mathrm{ml}$. Administramos a dose de $200 \mathrm{mg}$ duas vezes a três vezes nas 24 horas e pelo prazo de cinco dias. Registramos a taxa global de cura parasitológica de $90 \%$ (27 doentes) e excelente tolerância ao produto.
\end{abstract}

\section{INTRODUC̣ÃO}

A Nitrimidazina substância sintetizada por De Carneri (6) no Instituto de Pesquisas Terapêuticas Carlo Erba de Milão, através do Metronidazol, mostrou tanto "in vitro" como "in vivo" intensa atividade antiprotozoário.

Inicialmente, as pesquisas demonstraram ser a droga um potente tricomonicida $(1,4,8,16,17,20)$ e, posteriormente, também ficou constatado sua excelente ação no combate à infestação causada pela Giardia lamblia $(3,10,11,12,13,20,22)$.

Em nosso meio a giardíase íncide com muita frequêencia, adquirindo papel relevante na etiologia das diarréias, desnutrição, distrofias em crianças, e, por outro ladc, diversos trabalhos vieram mostrar que a Giardia lamblia pode provocar alterações duodenais e esteatorreia $12,5,7,9$, $14,15,18,21$ ).

Anteriormente, um de nós (D. H.) em ensaio inicial $(10,11)$ verificou excelente atividade da substância em tela no trata- mento da giardiase. Baseado em nossa experiência e na de outros AA., resolvemos desenvolver novos estudos com a droga em crianças portadoras de giardíase. É o que pretendemos relatar em nossa atual investigação.

\section{MATERIAL E MÉTODOS}

Tratamos 30 crianças de giardíase com o derivado Nitrimidazínico na apresentação de suspensão e divididas em dois grupos constituidos cada por 15 pacientes.

Grupo A - Neste grupo, tratamos 15 crianças matriculdadas nos Ambulatórios da Clínica de Doenças Infectuosas e Parasitárias da F.M.U.P.Pe., cujas idades variaram de dois a dez anos lum paciente respectivamente), enquanto os pesos oscilaram em torno de 8 a $26 \mathrm{~kg}$ rum doente) e oito eram do sexo feminino e sete do masculino. Empregamos a Nitrimidazina na forma de suspensão contendo 200 mg da substância base por cada $5 \mathrm{ml}$, na posologia diária de $400 \mathrm{mg}(10 \mathrm{ml})$ dividi-

Trabalho da Clinica de Doenças Infectuosas e Parasitarias e Disciplina de Puericultura e Clínica da Primeira Infancia da F.M.U.F.Pe.

* Prof. Adjunto

* * Auxiliar de Ensino.

Recebido para publicação em $1.36 .7 \%$ 
da em duas tomadas nas 24 horas, uma administrada após o desjejum e a última após o almoço, durante cinco dias consecutivos (dose total: 2,0g).

C diagnóstico da protozoose foi efetuado pelas técnicas de Faust e cols. e a de Hofman, Pons e Janer com sedimentação durante 24 horas. Para o controle de cura parasitológica realizamos os mesmos métodos laboratoriais com intervalos de oito e 25 dias após o tratamento.

As manifestaçōes clínicas apresentadas pelos doentes foram bastante proteiformes entretanto predominaram os seguintes sintomas: náuseas, vômitos, dor epigástrica, fezes líquidas ou pastosas, anorexia, emagrecimento e distensão abdominal.

Grupo B - Também constituído por 15 crianças matriculadas no Ambulatório da Disciplina de Puericultura e Clínica da $1^{*}$ Infância da F.M.U.F.Pe., com idades variáveis em torno de dez meses (um doente)) a oito anos (dois enfêrmos) e o peso entre oito (dois casos) a $23 \mathrm{~kg}$ (um paciente). Dez eram do sexo masculino e cinco do feminino; administramos a Nitrimidazina em suspensão (cada $5 \mathrm{ml}$ contém 200 mg da substância base) na dose diária de $200 \mathrm{mg}$ três vezes ao dia, dividida em três tomadas nas 24 horas, uma após cada refeição e durante cinco dias seguidos (dose total: $3,0 \mathrm{~g}$ ).

Para o diagnóstico e controle de cura parasitológica, submetemos os pacientes aos mesmos exames do Grupo A e a sintomatologia apresentada pelos enfermos foi praticamente a mesma incluindo ainda insônia, sono agitado e irritabilidade.

\section{RESULTADOS}

Obtivemos no Grupo A $80 \%$ de cura parasitológica (ver quadro) e todos os enfermos toleraram bem o medicamento, não se registrando nenhuma manifestação colateral. Observamos também melhora clinica após o tratamento em todos os doentes.

Nc Grupo B verificamos $100 \%$ de cura parasitológica e clínica, bem como excelente tolerância ao produto.

O resultado global de cura parasitológica obtida foi de $90 \%$, ou seja 27 pacientes.

\section{QUADRO}

Resultados obtidos com a Nitrimidazina no tratamento da giardiase.

$\begin{array}{cccc}\text { Grupos } & N^{\circ} \text { Casos } & \text { Dose Total } & \% \text { Cura } \\ \text { A } & 15 & 2,0 \mathrm{~g} & 80 \% \\ \text { B } & 15 & 3,0 \mathrm{~g} & 100 \%\end{array}$

\section{COMENTÁRIOS E CONCLUSÕES}

Em trabalhos anteriores, Huggins (10, 11) medicando 20 pacientes ( 13 adultos $\mathrm{e}$ sete crianças com a dose total de $6,0 \mathrm{~g}$ e $1,875 \mathrm{~g}$ respectivamente), obteve $100 \%$ de cura parasitológica e excelente tolerância.

$\mathrm{Na}$ mesma época, Salles e cols. (20) medicaram 27 enfêrmos adultos de ambos os sexos, portadores de giardíase com a Nitrimidazina na dose diária de $1,0 \mathrm{~g}$ (quatro comprimidos de $250 \mathrm{mg}$ ) e pelo período de cinco dias. Realizaram controle de cura parasitológica com exames de fezes em intervalos de 7,15 e 22 dias após o tratamento. Verificaram sucesso terapêutico em 24 dcentes $(88,8 \%)$ e ótima tolerância.

Baranski e cols. (3) trataram 61 doentes portadores de giardiase, utilizando a dose diária de $250 \mathrm{mg}$ para as crianças e $500 \mathrm{mg}$ para adultos, durante cinco dias. Obtiveram $100 \%$ de cura parasitológica e excelente tolerância à medicação.

Zingano e cols. (22) medicaram 15 crianças portadoras de giardíase com dois esquemas posológicos: pacientes até $10 \mathrm{~kg}-$ $250 \mathrm{mg}$ por dia durante seis dias (dose total: $1,5 \mathrm{~g}$ ) e com até $30 \mathrm{~kg}-500 \mathrm{mg}$. por dia e pelo mesmo prazo (dose total: $3,0 \mathrm{~g}$ ). Verificaram cura parasitológica em $100 \%$ dos pacientes tratados, perfeita tolerância à substância e melhora do estado geral dos doentes com desaparecimento da irritabilidade a acentuada melhora do apetite.

Levi e cols. (12) utilizaram a Nitrimidazina na forma de suspensão no tratamento de crianças portadoras de giardiase com vários esquemas terapêuticos $(200$ $\mathrm{mg}$ em duas ou três doses nas 24 horas, durante cinco ou sete dias); registraram a taxa global de cura parasitológica de $92 \%$ e a tolerância à droga foi satisfatória.

Em recente trabalho, Louzada e cols (13) realçaram a grande atividade da $\mathrm{Ni}$ trimidazina sobre a Giardia lamblia e sua excelente tolerabilidade, ao empregarem a substância em 69 pacientes. Utilizaram oito 
esquemas terapêuticos e concluíram oue a posoiogia total ótima para a obtençāo de $100 \%$ de cura parasitológica nāo deve ser inferior a $2,0 \mathrm{~g}$.
Pelc exposto e através da nossa experiência, concluímos ser o derivado Nitrimidazínico um potente giardicida e dotado de excelente tolerabilidade.

SUMMARY

The Authors treated 30 children with. Giardia lamblia infection. The doily corage employed was 400 to $600 \mathrm{mgm}$, during five days, and consequently $a$ total posology of $2,0 \mathrm{gm}$ and $3.0 \mathrm{gm}$.

The cure control was made by Hoffman, Pons and Janer technique rt the sth and 25 th ciays after treatment. The parasitological cure rate cbtrined was 50\% (27 pctients); the excellent tolerance to the drug was pointed out.

The Authors concluded that Nitrimidazine is a potent, atoxis, and well tolerated drug against Giardia lamblia injection.

\section{REFERENCIAS BIBLIOGRÁFICAS}

1 - ALMEIDA NETC. P. P GUIMARÃFS M. V. - Un novo trichomonicida. Hospital (Rio de Janeiro!, 77: 1.5811.585, 1970 .

2 - AMINI, F. - Giardiasis and steatcrrhoea. J. Trop. Med. Hyg., 66: 150192, 1964.

? - BARANSKI, M. C., GOMES, N. R., FRANCC DE GODOY, O.. SILVA. A. F., SPEITZER, N., KOTAKA, P. I. $e$ SILVEIRA, H. B. - Tratamento da giardiase com Nitrimidazina. Tribuna Médica, XIV (375): 36-40, 1971.

4 - BARROS, M. - Tratamento da trichcmoniase vaginal com novo medicamento (Nitrimidazina). Hcspital (Rio de Janeiro), 77: 2.023-2.027. 1970 .

5 - BRANDBORG, L. L., TANKERSLEY, C. B., GOTTLIEB. S. BARANCIK, M. and SARTOR, V. E. - Histological demcnstration of mucosal invasion by Giardir lrimblin in man. Gastroentclogy, 52: 143-150, 1967.

6 -DE CARNERI, I. - Antiprotozoan activity of Nitroimidazoles. ArzneimForsch. (Drug Res.1, 19: 382-386. 1965.

$7 \ldots$ DOUSI, J. Y., HAGHIGHI, L., KAVCUSSI. K. M. - Ducdenitis and Giardiasis. J. Trop. Med. Hyg., 72 : 284-285, 1969.
8 - EMANUELI, A e DE CARNERI, I Clinical trials with Nitrimidazine, a new systemic trichomonacide. 6 th international Congress of Chemotherapy, Tokio (Kyoto', August 10th tc: 15 th, 1969

8- HOSKINS, L. C., WINAWER, S. J. BROITMAN, S. A., GOTTLIEB, L. S., and ZAMCHECK, N. - Clinical giardiasis and intestinal malabsorption. Gastroenterology, 53: 265-279, 1967.

10 - HUGGINs, D. - Ensaio clínico com $c$ derivado Nitrimidazínico (Naxogin) no tratamento da giardiase. Hospital (Rio de Janeiro), 77: 2.053$2.066,1970$.

11 - HUGGINS, D. -- Ensaio clínico com c derivado Nitrimidazinico Naxogin, no tratamento da giardiase. (Nota prévia). Anticguia Medica (Colombia), 20: 351-354, 1970.

12 - LEVI, G. C. AMATO NETO, V. e CAMPOS, L. L. - Tratamento da giardiase por meio da Nitrimidazina. VII Congresso da Sociedade Brasileira de Medicina Tropical, Manaus, 1071 .

13 - LOUZADA, J. I. Z.. LOUZADA, T. Z. e DE CARLI, G. A. - A Nitrimidazina ne giardíase. Tribuna Médiea. XIV $(378) ; 49-58,1971$. 
14- MAESTAK, R. F. and LINDNER, A. Di. Radiolngy of the small inestine. W. B. Saunders Co. Fhiladelnhia. USA, 1970 .

15 ... WORECKI, R. and PARKER, I. G - Ultrastructural stidies of huinan Giardia lamblia and subjacent jejunal mucosal in a subject with steatorrhea. Gastroentelology, 52: 15i$16^{4}, 1967$.

16 - OLIVEIRA, A. M. B., DELASCIO, D. e OLIVEIRA. N. - Tratamento da vaginite por trichomonas. (Resultades com um novo composto imidazolico: a Nitrimidazina). Hospital (Rio de aneirol, 78: 199-215, 1970.

17 - OLIVEIRA, F. C. e LIMA, R. T. Tratamento da trichomoniase genital pela Nitrimidazina em doentes grávidas. Hospital (Rio de Janeiro). 78: 561-565. 1970.

18 OLMOS, A., OYARCE, R., DONCKE, G., BUIL, F:, BIEL, F. y TORREJON. $G$. Algunos aspectos clinicos y fi- sicpatclogicos de la lambliase (Giardiasis. Estuaios en pacientes adultos. Bol. Chileno Farasit., 23: $48-56$, 1968 .

IS - ROGNONI. V. E SIDERI. L. - Tratramentc delie vaginiti con particolare riguardc alie forme di patogenes) protozoaria: risultati con un ruovc oreparato Nitrimidazolico per uso lcuaie. Riv. Ost. Ginec. Prat. 51: $257,1968$.

20 -. SALLES, J. M. C., COSTA, C. A. E MATOS, L. F. -- Avaliacāo da atividade da Nitrimidazina na giardiase $e$ triccmoniase genital. Hospital (Rio de Janeiro), 77: 1.689--1.697, 1970.

21 - YARDLEY, J. $H$. and BAYLESS, T. M. - Giardiasis. Gastroenterology. 52: $301-304,1967$.

22 - ZINGANC, A. G.. FROES, O. M. e LIMA, D. F. - A Nitrimidazina no tratamentc da giardiase. Hospital Rio de Janeiro!. 79: 135-139, 1971. 